\section{The efectiveness of narrowband ultraviolet B (NB- UVB) in Vitiligo Management and its Association with Thyroid Stimulating Hormone (TSH) level in Indonesian population}

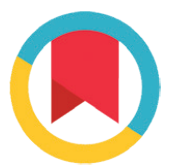

CrossMark

\author{
Farida Tabri*
}

\title{
Abstract
}

Objective: Vitiligo is an autoimmune disease marked by the presence of hypopigmented macules due to the reduction in the number and function of melanocytes. Besides its adverse cosmetic effect, vitiligo is also associated with other autoimmune diseases such as thyroid disorders. Ultraviolet irradiation, especially narrowband ultraviolet $B$ (NB-UVB), has been widely used in treating vitiligo despite the unclear mechanism. However, such studies in Indonesian population are very limited. This study aims to examine the efficacy of NB-UVB in vitiligo treatment and its effect of thyroid function in Indonesian population.

Material and Methods: Twenty-two vitiligo patients aged 4-54 years old were treated three times weekly with NB-UVB. The minimal erythema dose (MED) was determined and an initial dose of $50 \%$ MED dose was commenced three times per week. The dose was increased gradually while assessing for the treatment response. The change in depigmentation rate, vitiligo area severity index (VASI) and the level of thyroid stimulating hormone (TSH) were assessed before and after therapy using Wilcoxon test.

Results: Depigmentation rate and VASI score were found to decrease after treatment, although the change was not statistically siginificant. Two patients showed an decrease in depigmentation rate of more than $75 \%$. The TSH level significantly decreased after treatment ( $p>0.05$ ). The most common side effect found was the combination between erythema, soreness, and pruritus.

Conclusion: Our study shows that NB-UVB therapy results in improved depigmentation, VASI score and a reduced TSH level in vitiligo patients.
Department of Dermatology and Venereology, Faculty of Medicine, Hasanuddin University, Makassar, Indonesia
*Corresponding to: Farida Tabri, Department of Dermatology and Venereology, Faculty of Medicine, Hasanuddin University, Makassar, Indonesia

farida.tabri.dv@gmail.com

Received: 30 May 2018

Revised: 10 August 2018

Accepted: 7 November 2018

Available Online 1 December 2018

Keywords: Vitiligo, Narrowband UVB, Thyroid stimulating hormone

Cite this Article: Tabri F. 2018. The efectiveness of narrowband ultraviolet B (NB-UVB) in Vitiligo Management and its Association with Thyroid Stimulating Hormone (TSH) level in Indonesian population. Journal of Dentomaxillofacial Science 3(3): 149-151. D0I: 10.15562/jdmfs.v3i3.843

\section{Introduction}

Vitiligo is an acquired depigmentation disorder marked by the presence of hypopigmented macules due to the reduction in the number and function of melanocytes which may lead to significant adverse psychosocial impact. ${ }^{1,2}$ This condition is thought to be autoimmune-mediated where melanocyte defects drive an autoimmune response leading to melanocyte destruction and hypopigmented lesions. ${ }^{3}$

Besides its adverse cosmetic effect, some studies have also suggested that vitiligo is associated with other autoimmune diseases such as thyroid disorders. A recent meta-analysis found the prevalence of autoimmune thyroid disorders among patients with vitiligo was $14.3 \%{ }^{4}$

Despite the development of various surgical and non-surgical modalities, the end result is still often unsatisfactory. Physical treatment using ultraviolet irradiation has been widely used in treating vitiligo despite the unclear mechanism. Several options such as psoralen and ultraviolet A (PUVA), broadband ultraviolet $\mathrm{B}$ (BB-UVB), or narrowband ultraviolet B (NB-UVB) have been studied and compared in the recent years.
However, data on clinical experience of treating vitiligo using NB-UVB in Indonesia is still limited. Most studies available are mostly done in the western hemisphere and there are only scarce data available in eastern population. Thus, this study aims to examine the efficacy of NB-UVB in vitiligo treatment and its effect of thyroid function reflected by TSH level in Indonesian population.

\section{Material and Methods Study Design}

The study was conducted in the Dermatovenereology Department, Faculty of Medicine, Hasanuddin University from June 2017 until December 2017 and has obtained approval from the Institutional Ethical Review Board. This was a clinical trial using a one group pre-post trial which compared the result before and after treatment.

\section{Subjects}

A total of 22 subjects with Fitzpatrick skin type IV and $\mathrm{V}$ were included in this study. All patients with vitiligo aged above four years old were eligible for 
this study. Informed consent was obtained from each subject. In pediatric patients, consent was obtained from the parents. Patients with skin disorders responsive to phototherapy, currently taking photosensitizer drugs or corticosteroids, history of photosensitizing reactions, and pregnancy were excluded. The sampling method used was consecutive sampling.

\section{Study Protocol}

During the initial visit, the vitiligo area scoring index (VASI) and vitiligo activity disease score (VIDA) were recorded. Clinical photograph using a digital camera was also taken as a baseline value to assess the change in depigmentation rate. The minimal erythema dose (MED) was determined and an initial dose of 50\% MED was commenced

Table 1 Lesion Distribution

\begin{tabular}{lc}
\hline Body site & $\mathbf{N}(\%)$ \\
\hline Trunk & $1(4.5)$ \\
Trunk, lower extremities & $6(27.3)$ \\
Trunk, hand & $1(4.5)$ \\
Upper arm & $1(4.5)$ \\
Face & $2(9.1)$ \\
Face, trunk & $3(13.6)$ \\
Face, lower extremities & $1(4.5)$ \\
Face, neck & $2(9.1)$ \\
Face, neck, trunk & $1(4.5)$ \\
Face, neck, hand & $1(4.5)$ \\
Hand & $3(13.6)$ \\
Total & 22 \\
\hline
\end{tabular}

Table 2 Depigmentation and VASI score before and after treatment

\begin{tabular}{lccc}
\hline \multirow{2}{*}{ Parameter } & \multicolumn{2}{c}{ Mean } & \\
\cline { 2 - 3 } & Before & After & P-value \\
\hline Depigmentation & $90.23 \pm 54.22$ & $85.45 \pm 57.63$ & 0.180 \\
VASI Score & $6.37 \pm 6.34$ & $6.28 \pm 6.42$ & 0.180 \\
TSH & $2.52 \pm 1.43$ & $1.83 \pm 1.36$ & 0.014 \\
\hline
\end{tabular}

Table 3 Side effects

\begin{tabular}{lc}
\hline Side Effects & $\mathbf{N}(\%)$ \\
\hline Erythema & $7(31.8)$ \\
Soreness & $1(4.5)$ \\
Pruritus & $1(4.5)$ \\
Combination & $13(59.1)$ \\
Total & $22(100)$ \\
\hline
\end{tabular}

(Dermalight $1000^{\circ}$, Dr. Hoenle Inc). Areas other than the lesion site were covered by black polyester cloth. The eyes were covered with UV-blocking goggles. The TSH level before and after treatment was also measured.

The treatment was administered three times per week on non-consecutive days and the irradiation dose was increased by $20-30 \%$ in each subsequent visit. Clinical photograph was taken in each visit. If mild erythema and pruritus occurred, the irradiation dose was kept constant until the side effects disappeared. In case of significant burning pain, blistering, and erythema occurred, the irradiation dose was decreased by $15 \%$. Once a $75 \%$ decrease in depigmentation rate was achieved, the treatment frequency was decreased to two times per week for four weeks followed by once per week for the next four weeks.

At the end of the treatment, the clinical condition was compared with the initial condition to determine the change in depigmentation.

\section{Statistical Analysis}

The data in this study was analyzed using Statistical Package for Social Sciences (SPSS) 21.0 for Windows (SPSS Inc. Chicago, IL, USA). Results were presented in the form of tables and charts. Wilcoxon test was used to compare the mean in depigmentation rate, VASI score, and TSH level. A p-value of less than 0.05 was considered as significant.

\section{Results}

All 22 patients, 13 (59.1\%) were males and $9(40.9 \%)$ were females. Their ages ranged from 5-54 years with a mean age of 26 years. The duration of disease ranged from three months to 25 years with a mean of 8 years. Table 1 shows the site distribution of the lesion. Six subjects were shown to have lesions on the trunk and lower extremities.

One patient received NBUVB for nine sessions while the remaining 21 patients received NB-UVB for 36 sessions. Table 2 shows the depigmentation, VASI score, and TSH level before and after therapy. A $75 \%$ decrease in depigmentation rate was shown by two subjects. The VASI score was found to decrease in two patients. The mean TSH level was significantly reduced after treatment.

The total cumulative dose in this study was $1185.14 \pm 349.42 \mathrm{~mJ}(498 \mathrm{~mJ}-1972 \mathrm{~mJ})$. Side effects observed in this study were shown in table 3 . The most common side effect found was the combination between erythema, soreness and pruritus. 


\section{Discussion}

This study shows the prevalence of males affected with vitiligo is slightly higher compared to females $(59.1 \%$ vs $40.9 \%)$ respectively. Most epidemiological studies are in line with this result, showing that there is no significant difference between male and female in acquiring vitiligo, ${ }^{5,6}$ although another study show a slight female predominance. ${ }^{7}$ Our population also did not show any age preferences as it was distributed in a relatively even fashion among various age groups. Present data suggests that vitiligo may occur at any age.

The depigmentation and VASI score showed a decreasing trend after treatment, although this change is not statistically significant ( $\mathrm{p}>0.05)$. Some studies have suggested the efficacy of NB-UVB in vitiligo treatment. ${ }^{8}$ A study by Bansal et al. ${ }^{9}$ showed a reduction in VASI score following NB-UVB treatment. ${ }^{9}$ The mechanism of action of NB-UVB in vitiligo is not yet fully understood. It is postulated that NB-UVB might inhibit melanocyte destruction or stimulate melanocyte production and migratory capacity.

Although the change was not statistically significant, clinical examination showed an apparent decrease in depigmentation. Two subjects have shown a significant improvement $(>75 \%)$. We postulate that this trend might be more evident had the treatment been done for a longer duration of time. Studies have shown that NB-UVB is superior to Psoralen and Ultraviolet A (PUVA). A double-blind randomized controlled study showed $64 \%$ patients with more than $50 \%$ in the NB-UVB group compared to only $36 \%$ in PUVA group. $^{10}$

The level of TSH in this study was shown to significantly reduced after treatment with NB-UVB. Literatures have suggested the association between hypothyroidism, shown by increased TSH, and vitiligo. ${ }^{11,12}$ Vitiligo might precede thyroid dysfunction by many years before clinical manifestation occurs and hence early screening may prevent severe comorbidity. ${ }^{11}$ Recent molecular studies have reported the possibility of shared heritable susceptibility genes in both vitiligo and thyroid dysfunction. Genome-wide linkage analysis suggested nine loci potentially involved in autoimmune thyroid dysfunction and vitiligo. ${ }^{13,14}$

Side effects observed in this study were erythema, soreness, and pruritus. However, these effects were mild and did not necessitate treatment cessation. The good safety profile of NB-UVB is also shown by other studies. A trial by Kumar et al..$^{15}$ showed that only $7 \%$ of subjects in their study experienced mild erythema, burning, or pruritus and only $6 \%$ reported xerosis. ${ }^{15}$

\section{Conclusion}

Our study shows that NB-UVB therapy results in improved depigmentation, VASI score and a reduced TSH level in Indonesian vitiligo patients.

\section{Acknowledgment}

The author would like to thank all participants who have participated in this study.

\section{Conflict of Interest}

The author reports no conflict of interest.

\section{References}

1. Manga P, Elbuluk N, Orlow SJ. Recent advances in understanding vitiligo. F1000 Res 2016;5: 2234.

2. Amerio P, Tracanna M, De-Remigis P, et al. Vitiligo associated with other autoimmune diseases: polyglandular autoimmune syndrome types 3B+C and 4. CPD 2006;31: 746-749.

3. Rodrigues M, Ezzedine K, Hamzavi I, et al. New discoveries in the pathogenesis and classification of vitiligo. J Am Acad Dermatol 2017;77: 1-13.

4. Vrijman C, Kroon M, Limpens J, et al. The prevalence of thyroid disease in patients with vitiligo: a systematic review. Br J Dermatol 2012;167: 1224-1235.

5. Lu T, Gao T, Wang A, et al. Vitiligo prevalence study in Shaanxi Province, China. Int J Dermatol 2007;46: 47-51.

6. Patil S, Gautam M, Nadkarni N, et al. Gender differences in clinicoepidemiological features of vitiligo: a cross-sectional analysis. ISRN Dermatol 2014;2014: 186-197.

7. Zhang Y, Cai Y, Shi M, et al. The prevalence of vitiligo: a meta-analysis. PLoS One 2016;11: e0163806-e.

8. Dillon AB, Sideris A, Hadi A, et al. Advances in vitiligo: an update on medical and surgical treatments. JCAD 2017;10: 15-28.

9. Bansal S, Sahoo B, Garg V. Psoralen-narrowband UVB phototherapy for the treatment of vitiligo in comparison to narrowband UVB alone. Photodermatol Photoimmunol Photomed 2013;29: 311-317.

10. Yones SS, Palmer RA, Garibaldinos TM, et al. Randomized double-blind trial of treatment of vitiligo: efficacy of psoralen-UV-A therapy vs Narrowband-UV-B therapy. Arch Dermatol 2007;143: 578-584.

11. Gupta Y, Ammini AC. Vitiligo, hypothyroidism and cardiomyopathy. Indian J Endocrinol Metab 2012;16: 463-465.

12. Baldini E, Odorisio T, Sorrenti S, et al. Vitiligo and autoimmune thyroid disorders. Front Endocrinol (Lausanne) 2017;8: 290.

13. Jin Y, Birlea SA, Fain PR, et al. Genome-wide association analyses identify 13 new susceptibility loci for generalized vitiligo. Nat Genet 2012;44: 676.

14. Czajkowski R, Męcińska-Jundziłł K. Current aspects of vitiligo genetics. Adv Dermatol \& Allergol/Postępy Dermatol Alergol 2014;31: 247.

15. Kumar YHK, Rao GRR, Gopal K, et al. Evaluation of narrow-band UVB phototherapy in 150 patients with vitiligo. Indian J Dermatol, Venereol, Leprol 2009;75: 162.

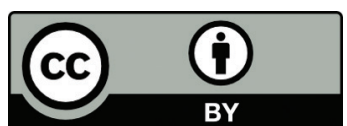

This work is licensed under a Creative Commons Attribution 